\title{
Breastfeeding and the risk of rotavirus diarrhea in hospitalized infants in Uganda: a matched case control study
}

Eric Wobudeya ${ }^{1,2^{*}}$, Hanifa Bachou ${ }^{1}$, Charles K Karamagi ${ }^{2}$, Joan N Kalyango ${ }^{2}$, Edrisa Mutebi ${ }^{3}$, Henry Wamani ${ }^{4}$

\begin{abstract}
Background: Rotavirus is responsible for over 25 million outpatient visits, over 2 million hospitalizations and 527,000 deaths annually, worldwide. It is estimated that breastfeeding in accordance with the World Health Organization recommendations would save 1.45 million children's lives each year in the developing countries. The few studies that examined the effect of breastfeeding on rotavirus diarrhea produced conflicting results. This study aimed to determine the effect of breastfeeding on rotavirus diarrhea among admitted infants in Uganda.

Methods: The study was conducted in the Pediatrics medical emergency unit of a National Referral hospital during a peak incidence time for rotavirus from February to April 2008. It was an age matched case-control study with a ratio of 1:1. We consecutively enrolled infants presenting at the study site during this period whose caretakers consented to participate in the study. A minimum sample size of 90 pairs was adequate with power of $80 \%$ to detect a $30 \%$ decrease in breastfeeding rate among the cases assuming a breastfeeding rate of $80 \%$ in the controls. The infants with rotavirus positive results were the "cases". We used the commercial enzyme immunoassay kit (DAKO IDEIATM rotavirus EIA detection kit) to diagnose the cases. The "controls" were admitted children with no diarrhea. We compared the cases and controls for antecedent breastfeeding patterns.

Results: Ninety-one matched case-control age-matched pairs with an age caliper of one month were included in the analysis. Breastfeeding was not protective against rotavirus diarrhea (OR 1.08: 95\% Cl $0.52-2.25 ; \mathrm{p}=0.8$ ) in the conditional logistic model.

Conclusions: Our study findings did not reveal breastfeeding as protective against rotavirus diarrhea in infants. This suggests searching for other complementary preventive methods such as rotavirus vaccination and zinc supplementation to reduce the problem of rotavirus diarrhea in infants irrespective of their feeding practices.
\end{abstract}

\section{Background}

Diarrhea is estimated to cause 1.5 million deaths and $21 \%$ of all under fives mortality worldwide [1]. It accounts for about five childhood deaths per 1000 population [2] mostly from developing countries. Annually Rotavirus diarrhea is associated with over 25 million outpatient visits, 2 million hospitalizations and 527,000 deaths per year worldwide [3]. Likewise, most of the under five diarrhea cases are caused by rotavirus diarrhea [4,5]. A study in Ghana found an incidence of 89

\footnotetext{
* Correspondence: ewobudeya@gmail.com

'Department of Paediatrics \& Child Health, Mulago National Referral Hospital. P. O. Box 7051 Kampala, Uganda

Full list of author information is available at the end of the article
}

diarrhea episodes per 1000 children per year of which 35 episodes were due to rotavirus diarrhea [6].

The Human rotavirus infection spreads by direct person-to-person contact. Effective hand washing and disposal or disinfection of contaminated items is therefore theoretically an important measure in the prevention of rotavirus infection.

Breastfeeding could reduce gastrointestinal infections as breast milk contains lactadherine, secretory IgA, T \& Blymphocytes, bactericidal lactoferrin, oligosaccharides [7] and human milk glycans $[8,9]$ that protect the intestinal epithelium against pathogens. The major component that is thought to prevent symptomatic rotavirus infection is lactadherine [10] while the anti-rotavirus antibodies in human milk seem to play a smaller role [11]. Although a

\section{Biomed Central}


study by Ray \& Kelkar found low maternal serum neutralizing antibody titers to rotavirus in mothers whose children were recovering from severe rotavirus disease suggesting increased predisposition [12], another study by Asensi et al that quantified the human milk rotavirus IgA antibodies did not demonstrate this correlation [11]. Breast milk anti-bodies to rotavirus has however been sited as one of the possible factors for the low rotavirus vaccine efficacy seen in the low income countries compared to the high income countries [13]. The current WHO guidelines on diarrhea management recommend continued breastfeeding during the diarrhea episode.

However, the specific role of breastfeeding in the prevention of rotavirus diarrhea has not been well established but it is generally considered to at least the reduce the severity of the disease [14]. A case control study by Clemens $J$ et al found that exclusive breastfeeding protects against rotavirus diarrhea in infants [15]. A prospective study by Naficy AB et al found a lower incidence of rotavirus diarrhea in infants that received breast milk [16]. A nested case control study by Dennehy PH et al found that breastfeeding in the previous month was protective in infants below 6 months [17]. Plenge-Bönig, A et al in a nested case control study in Europe of infants with acute gastroenteritis found breastfeeding to be protective against rotavirus gastroenteritis compared to other causes of gastroenteritis [18]. A review paper by Golding J et al did not find a protective effect of exclusive breastfeeding in infants aged 4 - 6 months against rotavirus diarrhea [19]. A prospective study by Misra SM et al found no difference in the rotavirus rates between the exclusively and non-exclusively breastfed infants [20]. The study may have not detected the difference because of the small number of 34 infants in the cohort studied. In a prospective study by Gurwith $M$ et al breastfeeding appeared not protective against rotavirus diarrhea [21]. This study of 104 infants and there 62 siblings was not designed to determine the relationship between breastfeeding and rotavirus diarrhea. A longitudinal study by Linhares A et al in children aged $0-3$ years in Brazil found no evidence of protection against clinical rotavirus disease by maternal milk [22].

The differences in study designs and age populations studied have led to variations in study results on effect of breastfeeding against rotavirus diarrhea. Therefore, we designed an age matched case-control study to investigate the effect of breastfeeding on rotavirus diarrhea among hospitalized Ugandan infants.

\section{Methods}

\section{Study design}

This was an age-matched case-control study with a ratio of $1: 1$. We matched controls within an age range of \pm 1 month of the cases.

\section{Study setting}

The study took place in the Pediatrics medical emergency unit of Mulago national referral hospital, Kampala, Uganda. This hospital also doubles as a primary health care centre for the surrounding areas. The Pediatric emergency unit of the hospital receives all children with severe medical conditions for overnight care. The children requiring further care transfer to one of the five main Pediatrics wards. The average monthly admission is about 1,000 children of whom about half of these are infants. About $7.1 \%$ of these infants have diarrhea with $45 \%$ due to rotavirus infection [23].

The definition of "Diarrhea" in this study was the passage of at least three loose or watery stools in any 24-hour period.

The cases consisted of infants admitted to the pediatric medical emergency unit of Mulago hospital with rotavirus diarrhea. The Controls were infants admitted to Pediatrics emergency unit of Mulago hospital with no diarrhea and had a negative stool sample for rotavirus. All parents/caregivers gave written informed consent to participate in the study. Caregivers are legal representatives of the participants other than the parents. We excluded infants with signs of respiratory tract infections and unknown feeding practices.

\section{Sampling procedure and matching}

A trained research assistant screened all the infants with diarrhea reporting at the Pediatric emergency unit registration desk for the study. We consecutively enrolled into the study those fulfilling the eligibility criteria. All the eligible children had their stools collected within 24 hours of admission to avoid nosocomial rotavirus infection. We batched the stool samples for a maximum of 1 week at $8^{\circ} \mathrm{C}$ before rotavirus analysis. A trained research assistant to match the available cases screened children with no diarrhea reporting at the ACU registration desk for eligibility as admitted controls. The controls also had their stools collected within 24 hours of admission and analyzed for rotavirus within seven days. The recruitment of both the cases and controls was concurrent.

If more than one age-matched control was eligible for matching a case, we choose the control by simple random sampling from the available eligible controls. We wrote the controls' identification numbers on pieces of paper, and by random sampling, one paper chosen. The control corresponding to the chosen identification number was then age-matched with the available case. We captured the information from the parents/caregivers onto a standardized semi-structured questionnaire. All the children with diarrhea received standard of care for diarrhea that included rehydration therapy and zinc supplementation by the attending physicians. 


\section{Measurements and data collection}

The research assistant not aware of the rotavirus status of the infants collected information about feeding practices. The main feeding practice of interest was whether the infant was breastfeeding or not. We defined an infant as "breastfed" if breast milk, either received directly from the breast or expressed, constitutes any portion of the infant diet. Feeding practice recall period was limited to 1 week prior to the interview. The socio-demographic data collected included: infant's age in months, caregivers or parents' age, education level and occupation. We measured the infants' weight and length using a hanging Salter scale and a measuring board, respectively. We recorded the weight to the nearest $10 \mathrm{~g}$ and length to the nearest 10 $\mathrm{cm}$. The research assistant inserted a size- 8 feeding tube into the infant's rectum and used a $5 \mathrm{ml}$-syringe to aspirate at least three milliliters of the stool. A laboratory runner transferred the sample to the laboratory in a screw caped container within a maximum of 60 minutes of collection. A competent laboratory technologist trained in rotavirus identification tested the samples for rotavirus antigen using a commercial enzyme immunoassay kit (DAKO IDEIA $^{\mathrm{TM}}$ rotavirus EIA detection kit) according to the standard operating procedures. The main outcome measure was the presence or absence of rotavirus antigens in the diarrhea stools of the study subjects.

\section{Data management and statistical analysis}

We captured the data in EpiData v3.1 (The EpiData Association, Odense, Denmark) and analyzed using STATA v9.2 (Stata, College Station, TX, USA).

We used the computer-based command for sample size for matched case control studies using Stata version 9.2 to calculate sample size. A sample size of 90 cases and 90 matched controls was estimated to detect a thirty percent difference in the breastfeeding rates between the cases and controls assuming a prevalence of breastfeeding of $80 \%$ in the controls with an accepted type 1 error of $5 \%$ ( $\alpha=0.05$ two-sided) and minimum power of $80 \%$.

We carried out Bivariate analysis comparing the predictors and the outcome in the matched cases and controls using Mantel Haenszel method. We used backward conditional logistic regression to assess for independent predictors. The effect measure was matched odds ratio (conditional OR). We used Ninety-five percent testbased confidence intervals (CIs) for the odds ratio. In order to control for the extraneous variables on the relationship between breastfeeding and rotavirus diarrhea, we used the conditional logistic regression model. The level of statistical significance was $\mathrm{p}<0.05$.

\section{Ethical issues}

We obtained Ethical approval from the Makerere university research and ethics committee before conducting the study. We obtained Written Informed consent from all the parents/caregivers.

\section{Results \\ Description}

The study took place in the Pediatrics medical emergency unit of a National Referral hospital during a peak incidence time for rotavirus from February to April 2008.

We screened two-hundred fifty (250) infants with acute diarrhea between February and April 2008. We excluded two infants due to unknown feeding practice and one hundred fifty seven had rotavirus negative diarrhea. Ninety-one stools were rotavirus positive. The 91 cases that were agematched with the 91 controls were included in the analysis. The baseline characteristics between the cases and controls were similar except for the sex (see Table 1). The mean maternal age for the cases and controls was 24.2 (SD 5) and 24.4(SD 5.2) years respectively but this difference was not statistically significant ( $p=0.7$ student's t test).

The majority $(70 \%)$ of our study infants were above the age of 6 months. The proportion of infants breastfeeding was $85 \%$ and $82 \%$ in the below 6 months and the above 6 months respectively. This difference was not statistically significant $(\mathrm{p}=0.5)$. Among infants

Table 1 Some socio-demographic characteristics of 91 rotavirus cases and 91 controls in Kampala, Uganda

\begin{tabular}{|c|c|c|c|c|}
\hline & & Cases & Controls & $P$ value \\
\hline \multicolumn{5}{|l|}{ Matching criteria } \\
\hline $\begin{array}{l}\text { Age (months), } \\
\text { mean (sd) }\end{array}$ & & $7.6(2.7)$ & $7.5(2.6)$ & 0.86 \\
\hline Characteristics & & n (\%) & n (\%) & \\
\hline \multirow[t]{2}{*}{ Sex } & Female & $27(29.7)$ & $41(45.1)$ & 0.032 \\
\hline & Male & $64(70.3)$ & $50(54.9)$ & \\
\hline \multirow{2}{*}{$\begin{array}{c}\text { Wasting }^{\mathrm{a}} \\
(\mathrm{Z} \text { score } \leq-2)\end{array}$} & Yes & 15 (16.6) & 16 (17.6) & 0.87 \\
\hline & No & $75(83.3)$ & 75 (82.4) & \\
\hline \multirow[t]{2}{*}{$\begin{array}{l}\text { Maternal } \\
\text { education }\end{array}$} & $\begin{array}{c}\text { Secondary \& } \\
\text { above }\end{array}$ & $45(49.4)$ & 49 (53.8) & 0.55 \\
\hline & $\begin{array}{c}\text { Below } \\
\text { secondary }\end{array}$ & $46(50.6)$ & $42(46.2)$ & \\
\hline \multirow{2}{*}{$\begin{array}{c}\text { Maternal } \\
\text { occupation }^{a}\end{array}$} & housewife & $59(66.3)$ & $53(58.8)$ & 0.3 \\
\hline & others & $30(33.7)$ & $37(41.1)$ & \\
\hline \multirow[t]{2}{*}{ Crowding ${ }^{b}$} & Yes & $28(30.8)$ & $25(27.5)$ & 0.62 \\
\hline & No & $63(69.2)$ & $66(72.5)$ & \\
\hline \multirow[t]{2}{*}{ Stool disposal } & Improper & $11(12.1)$ & $9(9.9)$ & 0.63 \\
\hline & Proper & 80 (87.9) & $82(90.1)$ & \\
\hline
\end{tabular}

$\mathrm{OR}$ indicates odds ratio. $\mathrm{Cl}$ indicates confidence interval.

${ }^{a}$ maternal occupation of 2 controls and 1 case were unknown; wasting data missing in 1 case.

${ }^{\mathrm{b}} 4$ or more per room. 
below 6 months, $77 \%$ of the cases and $92 \%$ of the controls were breastfeeding. This difference was not statistically significant $(\mathrm{p}=0.1)$. Among infants above 6 months, $56 \%$ of the cases and $76 \%$ of the controls were breastfeeding. This difference was not statistically significant $(p=0.09)$. The breastfeeding rates were similar between the cases and the controls (see Table 2).

\section{Breastfeeding and rotavirus diarrhea}

On matched bivariate analysis, breastfeeding was not associated with rotavirus diarrhea (Table 2). Complementary, exclusive and predominant breastfeeding were also not associated with rotavirus diarrhea (Table 2). However, this study did not have power to evaluate the various modes of breastfeeding.

The other factors associated with rotavirus diarrhea on matched bivariate analysis included vomiting, fever and sex (Table 2).

Sex and breastfeeding were included in the conditional logistic regression analysis. Vomiting and paternal education were not included because of significant missing data.

At multivariate analysis, using conditional logistic regression, breastfeeding was not protective OR 1.08 (95\% CI 0.52 - 2.25) against rotavirus diarrhea in infants after controlling for sex (Table 3). There was no interaction between breastfeeding and other factors.

\section{Discussion}

This was a hospital based age-matched case-control study. The study was conducted on the assumption that breastfeeding is still being practiced in Uganda in accordance with cultural norms and recommendations from the WHO as an intervention to reduce the incidence and severity of diarrhea disease. However, its protective role against rotavirus is not universally accepted. The observation that the diarrhea rates and the median age of rotavirus disease are not delayed in countries with prolonged versus short durations of breastfeeding brings into question the protective role of breastfeeding against rotavirus diarrhea. This study aimed to investigate the relationship between breastfeeding and rotavirus diarrhea.

The results of this study did not demonstrate the protective effect of breastfeeding against rotavirus diarrhea in infants. Previous reports have concurred on this topic $[21,24,25]$ in which none demonstrated significant overall protection of breastfeeding against rotavirus diarrhea. Duffy and Byers et al [14] followed a cohort of 197 infants through a winter season and found no difference in the rotavirus rates between the breast-fed and bottlefed infants. Gurwith and Wenman et al [21] in a follow up study of 104 infants for 16.3 months found no difference in the rotavirus rates between the breastfed and non-breastfed infants. A nested matched case-control study by Weinberg et al [24] of 50 infants found no difference in the breastfeeding rates between infants with and without rotavirus diarrhea. An exploratory study by Glass et al [25] out of the surveillance data in Dhaka Bangladesh found higher rates of rotavirus among breastfed infants hence questioning the protective role

Table 2 Unadjusted association between some factors and rotavirus diarrhea in 91 matched case-control pairs in Kampala, Uganda

\begin{tabular}{|c|c|c|c|c|c|}
\hline Variable & ${ }^{1}$ Cases (\%) & Controls (\%) & ${ }^{2} \mathrm{OR}$ & $95 \% \mathrm{Cl}$ & p-value \\
\hline Crowding (4 or more per room) & 31 & 27 & 1.1 & $0.6,2.3$ & 0.7 \\
\hline Sex (Male) & 70 & 55 & 1.8 & $1.02,3.4$ & 0.04 \\
\hline${ }^{\mathrm{a}}$ Febrile (axilla $\mathrm{T} \geq 37.5^{\circ} \mathrm{C}$ ) & 44 & 59 & 0.5 & $0.28,1.003$ & 0.05 \\
\hline bomiting & 85 & 35 & 20.5 & $4.9,84.7$ & $<0.001$ \\
\hline breastfeeding & 85 & 81 & 1.2 & $0.5,2.4$ & 0.5 \\
\hline Complementary breastfeeding & 66 & 59 & 1.3 & $0.7,2.5$ & 0.3 \\
\hline Exclusive breastfeeding & 12 & 19 & 0.5 & $0.1,1.3$ & 0.2 \\
\hline Predominant breastfeeding & 7 & 3 & 2 & $0.5,7.9$ & 0.5 \\
\hline${ }^{\mathrm{C}}$ Breastfeeding in Age $>6$ Months & 56 & 77 & 1.8 & $0.7,4.4$ & 0.15 \\
\hline${ }^{\mathrm{d}}$ Breastfeeding in Age $=<6$ Months & 77 & 92 & 0.3 & $0.06,1.6$ & 0.17 \\
\hline Improper disposal & 12 & 10 & 1.2 & $0.4,3.1$ & 0.8 \\
\hline Wasted $(\leq-2 \mathrm{Z})$ & 17 & 18 & 0.9 & $0.4,2.0$ & $>0.999$ \\
\hline Secondary education and above & 49 & 54 & 0.8 & $0.4,1.5$ & 0.6 \\
\hline Housewife & 66 & 60 & 1.2 & $0.6,2.3$ & 0.5 \\
\hline
\end{tabular}

${ }^{1}$ Proportion exposed to factor in case and control groups.

${ }^{2}$ Odds ratio by Mantel-Haenszel method.

${ }^{a} 85$ pairs were analyzed. Not powered enough.

${ }^{\mathrm{b}} 78$ pairs were analyzed. This analysis variable is not powered enough.

${ }^{\mathrm{C}} 70$ pairs were analyzed. This analysis variable not powered enough.

${ }^{d} 20$ pairs were analyzed. This analysis variable is not powered enough. 
Table 3 Adjusted association between breastfeeding and rotavirus diarrhea in $\mathbf{9 1}$ matched case-control pairs in Kampala, Uganda

\begin{tabular}{lcll}
\hline Variable & ${ }^{\xi}$ OR & $\mathbf{9 5 \% ~ C l}$ & p-value \\
\hline Breastfeeding (yes) & 1.08 & $0.52,2.25$ & 0.82 \\
Sex (Male) & 1.86 & $1.0,3.42$ & 0.048 \\
\hline
\end{tabular}

Odds ratio by Mantel-Haenszel method.

of breastfeeding. In a case-control study conducted by Clemens et al [15] in Bangladesh with hospital cases and community controls, breastfeeding was not protective against rotavirus diarrhea in infancy. This study may have not had the power to detect this difference given the infrequency of non-breastfed infants in the studied population. The close linkage between age, breastfeeding and rotavirus diarrhea could probably explain the observations from these reports. The peak age for rotavirus diarrhea is 6-11 months while the rates of breastfeeding begin to decline after 6 months. The protective effects of breastfeeding seem to wane with age [15]. This might be the reason why findings from the studies where the focus was on infants of 6 months and below showed a tendency to protection by exclusive breastfeeding. In our study, the majority of the study participants were over 6 months of age. The differences in the methodologies and the various definitions of breastfeeding make the interpretation and comparisons of these studies less precise. The assumptions of our study may have not enabled us to detect the observed fifteen percent difference in the breastfeeding rates between the cases and the controls.

A sub-analysis report by Clemens et al focusing on infants of 6 months or less showed a strong association between breastfeeding and rotavirus diarrhea. This report is however not reliable given the very low rates of exclusively breastfed infants in the study. The exploratory analysis from our study showed a tendency to protection from rotavirus by exclusive breastfeeding mostly in infants below 6 months but was not powered enough to draw any conclusions. Dennehy, PH et al [17] showed a protective role of breastfeeding against hospitalization due to rotavirus diarrhea. Our study result may have differed because we measured current breastfeeding and not breastfeeding in the previous month, and our controls were hospitalized children. PlengeBonig, A et al [18] has showed a protective role of breastfeeding in infants with rotavirus acute gastroenteritis compared to other causes of gastroenteritis. Our study results may have differed from this work because none of our controls had diarrhea.

To our knowledge, there is scarcely any published data on the relationship between rotavirus and exclusive breastfeeding in infants less than 6 months.
The intestinal mucosa may need continuous bathing with antibodies and other anti-infective components in breast milk for protection against rotavirus. This implies that sporadic or low volume feeds may be ineffective. This observation has been made by Ebina [26] and Berger [27] where infants who were fed on appropriate volume of milk with rotavirus antibodies were protected or had reduced severity of rotavirus diarrhea. The possibility exists that breastfeeding may be protective only if it is practiced with the intensity and frequency that allows continuous high protection of the mucosa rather than the sporadic small volumes. Besides Hjelt $\mathrm{K}$ et al [28] showed that the levels of secretory IgA antibodies to rotavirus are highest in colostrums and early breast milk and rapidly decline in the first few weeks of life. The low vaccine efficacy found in Africa and Asia $[29,30]$ might be explained by the early vaccination age and therefore the possibility of high vaccine interference by the higher levels breast milk antibodies [13].

In our study, sixty-three percent of the feeding practice was complementary breastfeeding. The factors determining the feeding practices are not random between the breastfed and non-breastfed infants. We may therefore be observing the impact of the complementary feeds on the risk of rotavirus diarrhea rather than the breastfeeding itself. Glass R et al [25] made a similar observation in the study where he observed an increased risk to rotavirus in infants 6-11 months and ninety percent of these children were on complementary breastfeeding. The observation in our study that none of the controls had a household contact with diarrhea supports the contagiousness of rotavirus within the home [31]. In our study, other factors such maternal education level, wasting in the infants and the methods of stool disposal did not influenced the relationship between breastfeeding and the risk of rotavirus diarrhea. The results from our study suggest that it may not just be breastfeeding but the mode of breastfeeding that determines its benefits. The protective effect of breastfeeding against rotavirus diarrhea has mainly been found in infants of 6 months and below [15]. These results also suggest that the observed non-benefit of breastfeeding against rotavirus may be due to environmental and sanitation factors rather than the breastfeeding itself. This therefore suggests that consideration of the background factors is critical in the realization of the benefits of breastfeeding on rotavirus diarrhea. Given the overall benefits of breastfeeding especially against bacterial diarrheal diseases, exclusive breastfeeding should be encouraged.

The study was limited by using hospital controls that usually are not representative of the community controls for the cases. The hospital controls are a highly selected group with unique and diverse background factors that 
do not represent the neighborhood factors among the cases. We however endeavored to limit this effect on our major outcome factor by excluding controls with conditions such as respiratory infections that are associated with breastfeeding. Our study design was unable to directly measure risk of rotavirus since the breastfeeding (exposure) and the outcome (rotavirus diarrhea) were measured at the same time. We reduced recall time for the feeding practice to one week in order to limit recall bias. If breastfeeding truly protects against only small doses of rotavirus diarrhea, then only cases with large inoculums may have developed severe disease requiring admission and therefore been included in our study. Our cases are a selected group of the severe forms of rotavirus infection. They may not be representative of the major pool of community rotavirus cases. We did age matching to cater for the differences in immunological responses and breastfeeding practice that change with age.

The study was about widely promoted concepts of breastfeeding and diarrhea in the public health messages. There is therefore the possibility that the responses from parents/caregivers were tailored to suit the health workers' expectations. We asked open questions concerning the feeding practices rather than whether the infants were breastfeed or not. We were however not able to verify the feeding practice in the infants. The majority of our study participants were above 6 months in whom the contribution of breastfeeding and supplemental feeding on rotavirus diarrhea could not be determined. If the intensity and frequency of breastfeeding influences the outcome of rotavirus, this study was not able to measure the intensities of breastfeeding between the cases and the controls.

We believe that these limitations did not significantly affect the results of this study.

\section{Conclusions}

Our study findings failed to show breastfeeding as protective against rotavirus diarrhea in infants. Since most of our study participants were above six months of age and on complimentary feeding, we recommend another study particularly focusing on breastfeeding and rotavirus diarrhea in the first 6 months of life.

\section{Acknowledgements}

We thank Moses, Jane and Augustine who were part of the research team. Moses and Jane were involved in the data collection. Augustine was the laboratory technologist who did the rotavirus identification.

We acknowledge the Uganda national Rotavirus surveillance Network that performed the Rotavirus testing.

The study was funded from the researchers' private funds.

\section{Author details}

${ }^{1}$ Department of Paediatrics \& Child Health, Mulago National Referral Hospital. P. O. Box 7051 Kampala, Uganda. ${ }^{2}$ Clinical Epidemiology unit, School of
Medicine. Makerere University College of Health sciences. P. O. Box 7062 Kampala, Uganda. ${ }^{3}$ Department of Internal Medicine, faculty of Medicine. Makerere University. P. O. Box 7062 Kampala, Uganda. ${ }^{4}$ School of Public Health Makerere University College of Health Sciences. P. O. Box 7062 Kampala, Uganda.

\section{Authors' contributions}

WE: conceived the study design, contributed substantially to the acquisition of data, carried out the statistical analysis, interpreted the data and drafted the manuscript. $\mathrm{BH}$ : contributed to the study design and was involved in revising the manuscript. KKC: contributed to the refining of the study design, interpretation of results and drafting of the manuscript. NKJ: contributed to the study design and critical revision of the manuscript. ME: contributed to the study design and the manuscript revision. $\mathrm{WH}$ : participated in the study design and has been critically involved in revising the manuscript. All authors read and approved the final manuscript.

\section{Competing interests}

The authors declare that they have no competing interests.

Received: 7 July 2010 Accepted: 17 February 2011

Published: 17 February 2011

\section{References}

1. WHO: Global Burden of Disease: 2004 update. Geneva: World Health Organisation; 2008

2. Kosek M, Bern C, Guerrant RL: The global burden of diarrhoeal disease, as estimated from studies published between 1992 and 2000. Bull World Health Organ 2003, 81(3):197-204.

3. WHO: Weekly epidemiological record. 2007, 32:285-296

4. Tswana SA, Mitchell JO, Richards PD: Detection of rotavirus in children under five years of age presenting with diarrhoea at Mapulaneng Hospital at Bushbuckeridge, Republic of South Africa. Cent Afr J Med 1998, 44(12):305-307

5. Haffejee IE, Moosa A: Rotavirus studies in Indian (Asian) South African infants with acute gastro-enteritis: I. Microbiological and epidemiological aspects. Ann Trop Paediatr 1990, 10(2):165-172.

6. Binka FN, Anto FK, Oduro AR, Awini EA, Nazzar AK, Armah GE, Asmah RH, Hall AJ, Cutts F, Alexander N, et al: Incidence and risk factors of paediatric rotavirus diarrhoea in northern Ghana. Trop Med Int Health 2003, 8(9):840-846

7. Morrow AL, Ruiz-Palacios GM, Altaye M, Jiang X, Guerrero ML, MeinzenDerr JK, Farkas T, Chaturvedi P, Pickering LK, Newburg DS: Human milk oligosaccharides are associated with protection against diarrhea in breast-fed infants. J Pediatr 2004, 145(3):297-303.

8. Newburg DS, Ruiz-Palacios GM, Morrow AL: Human milk glycans protect infants against enteric pathogens. Annu Rev Nutr 2005, 25:37-58.

9. Morrow AL, Ruiz-Palacios GM, Jiang $X$, Newburg DS: Human-milk glycans that inhibit pathogen binding protect breast-feeding infants against infectious diarrhea. J Nutr 2005, 135(5):1304-1307.

10. Newburg DS, Peterson JA, Ruiz-Palacios GM, Matson DO, Morrow AL, Shults J, Guerrero ML, Chaturvedi P, Newburg SO, Scallan CD, et al: Role of human-milk lactadherin in protection against symptomatic rotavirus infection. Lancet 1998, 351(9110):1160-1164.

11. Asensi MT, Martinez-Costa C, Buesa J: Anti-rotavirus antibodies in human milk: quantification and neutralizing activity. J Pediatr Gastroenterol Nutr 2006, 42(5):560-567.

12. Ray PG, Kelkar SD: Prevalence of neutralizing antibodies against different rotavirus serotypes in children with severe rotavirus-induced diarrhea and their mothers. Clin Diagn Lab Immunol 2004, 11(1):186-194.

13. Nelson EA, Glass RI: Rotavirus: realising the potential of a promising vaccine. Lancet 376(9741):568-570.

14. Duffy LC, Byers TE, Riepenhoff-Talty M, La Scolea LJ, Zielezny M, Ogra PL: The effects of infant feeding on rotavirus-induced gastroenteritis: a prospective study. Am J Public Health 1986, 76(3):259-263.

15. Clemens J, Rao M, Ahmed F, Ward R, Huda S, Chakraborty J, Yunus M, Khan MR, Ali M, Kay B, et al: Breast-feeding and the risk of lifethreatening rotavirus diarrhea: prevention or postponement? Pediatrics 1993, 92(5):680-685.

16. Naficy AB, Abu-Elyazeed R, Holmes JL, Rao MR, Savarino SJ, Kim Y, Wierzba TF, Peruski L, Lee YJ, Gentsch JR, et al: Epidemiology of rotavirus 
diarrhea in Egyptian children and implications for disease control. Am J Epidemiol 1999, 150(7):770-777.

17. Dennehy PH, Cortese MM, Begue RE, Jaeger JL, Roberts NE, Zhang R, Rhodes P, Gentsch J, Ward R, Bernstein DI, et al: A case-control study to determine risk factors for hospitalization for rotavirus gastroenteritis in U.S. children. Pediatr Infect Dis J 2006, 25(12):1123-1131.

18. Plenge-Bonig A, Soto-Ramirez N, Karmaus W, Petersen G, Davis S, Forster J: Breastfeeding protects against acute gastroenteritis due to rotavirus in infants. Eur J Pediatr 169(12):1471-1476.

19. Golding J, Emmett PM, Rogers IS: Gastroenteritis, diarrhoea and breast feeding. Early Hum Dev 1997, 49 Suppl:S83-103.

20. Misra SM, Sabui TKM, Basu SM, Pal NM: A Prospective Study of Rotavirus Diarrhea in Children Under 1 Year of Age. Clin Pediatr (Phila) 2007, 46(8):683-688.

21. Gurwith $M$, Wenman $W$, Hinde D, Feltham S, Greenberg H: A prospective study of rotavirus infection in infants and young children. $J$ Infect Dis 1981, 144(3):218-224.

22. Linhares AC, Gabbay YB, Freitas RB, da Rosa ES, Mascarenhas JD, Loureiro EC: Longitudinal study of rotavirus infections among children from Belem, Brazil. Epidemiol Infect 1989, 102(1):129-145

23. Nakawesi JS, Wobudeya E, Ndeezi G, Mworozi EA, Tumwine JK: Prevalence and factors associated with rotavirus infection among children admitted with acute diarrhea in Uganda. BMC Pediatrics 2010, 10(69).

24. Weinberg RJ, Tipton G, Klish WJ, Brown MR: Effect of breast-feeding on morbidity in rotavirus gastroenteritis. Pediatrics 1984, 74(2):250-253.

25. Glass RI, Stoll BJ, Wyatt RG, Hoshino Y, Banu H, Kapikian AZ: Observations questioning a protective role for breast-feeding in severe rotavirus diarrhea. Acta Paediatr Scand 1986, 75(5):713-718.

26. Ebina T, Sato A, Umezu K, Ishida N, Ohyama S, Ohizumi A, Aikawa K, Katagiri S, Katsushima N, Imai A, et al: Prevention of rotavirus infection by cow colostrum antibody against human rotaviruses. Lancet 1983, 322(8357):1029-1030.

27. Berger R, Hadziselimovic F, Just M, Reigel P: Effect of feeding human milk on nosocomial rotavirus infections in an infants ward. Dev Biol Stand 1983, 53:219-228.

28. Hjelt KGPC, Nelsen OH, Schiotz PO, Krasilnikoff PA: Rotavirus antibodies in the mother and her breastfed infant. J Pediatr Gastroenterol Nutr 1985, 4:414-420.

29. Armah GE, Sow SO, Breiman RF, Dallas MJ, Tapia MD, Feikin DR, Binka FN, Steele AD, Laserson KF, Ansah NA, et al: Efficacy of pentavalent rotavirus vaccine against severe rotavirus gastroenteritis in infants in developing countries in sub-Saharan Africa: a randomised, double-blind, placebocontrolled trial. Lancet 376(9741):606-614.

30. Zaman K, Dang DA, Victor JC, Shin S, Yunus M, Dallas MJ, Podder G, Vu DT, Le TP, Luby SP, et al: Efficacy of pentavalent rotavirus vaccine against severe rotavirus gastroenteritis in infants in developing countries in Asia: a randomised, double-blind, placebo-controlled trial. Lancet 376(9741):615-623

31. Dennehy PH: Transmission of rotavirus and other enteric pathogens in the home. Pediatr Infect Dis J 2000, 19(10 Suppl):S103-105.

\section{Pre-publication history}

The pre-publication history for this paper can be accessed here: http://www.biomedcentral.com/1471-2431/11/17/prepub

doi:10.1186/1471-2431-11-17

Cite this article as: Wobudeya et al: Breastfeeding and the risk of rotavirus diarrhea in hospitalized infants in Uganda: a matched case control study. BMC Pediatrics 2011 11:17.

\section{Submit your next manuscript to BioMed Central and take full advantage of:}

- Convenient online submission

- Thorough peer review

- No space constraints or color figure charges

- Immediate publication on acceptance

- Inclusion in PubMed, CAS, Scopus and Google Scholar

- Research which is freely available for redistribution 